\title{
L'observatoire des pratiques et des métiers de la presse
}

Philippe Loquay

\section{(2) OpenEdition}

1 Journals

Édition électronique

URL : http://journals.openedition.org/communicationorganisation/1748

DOI : 10.4000/communicationorganisation. 1748

ISSN : 1775-3546

Éditeur

Presses universitaires de Bordeaux

Édition imprimée

Date de publication : 1 novembre 1994

ISSN : 1168-5549

Référence électronique

Philippe Loquay, «L'observatoire des pratiques et des métiers de la presse », Communication et organisation [En ligne], 6 | 1994, mis en ligne le 26 mars 2012, consulté le 21 septembre 2020. URL: http://journals.openedition.org/communicationorganisation/1748; DOI : https://doi.org/10.4000/ communicationorganisation. 1748

Ce document a été généré automatiquement le 21 septembre 2020.

(c) Presses universitaires de Bordeaux 


\title{
L'observatoire des pratiques et des métiers de la presse
}

\author{
Philippe Loquay
}

1 La mise en place d'un Observatoire des pratiques de la presse était dans de nombreux esprits depuis quelques années. La multiplication des colloques sur le thème de l'éthique de l'information ${ }^{1}$, la réflexion menée par le Groupe de Carcans ${ }^{2}$ en fournissent des exemples. La question était de savoir si la profession de journaliste était prête a se prêter à l'analyse et comment Accepterait-elle en particulier d'aborder les problèmes déontologiques liés à ses propres pratiques avec des membres d'autres confréries que la sienne (chercheurs, juristes...) voire des représentants de la société civile (lecteurs, auditeurs ou téléspectateurs, enseignants...).

2 Une réponse est apportée par la création de l'Observatoire des pratiques et des métiers de la presse créé par le Centre de formation et de perfectionnement des journalistes (CFPJ) de Paris ${ }^{3}$ en septembre dernier.

3 Celui-ci s'est donné une triple vocation :- observer en continu l'évolution des pratiques et du métier de journaliste ; - animer la réflexion à ce sujet ; - produire des études et des enquêtes à son endroit.

4 L'observation consistera en la « collecte d'informations et de résultats de recherches au sein des équipes et des laboratoires du CNRS, de l'INA, etc, et des universités. Un échange est engagé avec plusieurs pays européens voisins: la Grande-Bretagne (Université de Stirling), la Belgique (Université de Louvain-la-Neuve), etc. L'Observatoire collaborera à l'enquête engagée par l'Association européenne de formation au journalisme, sur la question des transformations de la profession dans les différents pays des écoles adhérentes. » L'Observatoire publiera annuellement un « état de la profession... (qui).-fera le point sur les données statistiques de base et proposera un recensement de l'ensemble des travaux réalisés sur ce sujet en France. Il sera disponible sur support imprimé et électronique $»^{4}$

5 «L'animation de la réflexion sur les évolutions des pratiques et métiers de la presse prendra la forme de rendez-vous réguliers permettant aux journalistes, chercheurs et universitaires de se rencontrer et d'échanger autour de leurs travaux, ainsi que de 
débattre des questions d'actualité. $\aleph^{5}$ Un séminaire mensuel et des journées d'études sont prévus.

1991 ; L'Etat des médias. Paris, La Découverte, 1991 ; Cartes de presse. Enquête sur les journalistes, Paris, Stock, 1993 Jean-Marie Charon a été rédacteur en chef de la revue Média-pouvoirs de 1988 à

7 1992. Ili coordonné avec Jean-Clément Texier l'étude comparative sur les quotidiens nationaux français et leurs homologues européens pour le compte du SPI'et du SJTI

Enfin, «à la demande d'entreprises et d'organismes publics et privés, l'Observatoire réalisera des études et recherches. Ces études devront être en rapport avec le journalisme, l'information, l'évolution des médias, le lectorat, etc. Ces travaux ont vocation à être pluridisciplinaires. Ils bénéficieront de la diversité des compétences présentes au CFPJ, ainsi que de la possibilité de constituer des équipes complémentaires en France, comme en Europe. $»^{6}$

9 La responsabilité de cet Observatoire a été confiée à Jean-Marie Charon, docteur en sociologie et ingénieur au CNRS, auteur de plusieurs ouvrages sur les médias et le journalisme. ${ }^{7}$

10 On peut finalement se poser la question de savoir si cet Observatoire saura dépasser les simples intérêts immédiats d'un centre de formation qui cherche à renforcer son influence, soumis à la concurrence de l'école supérieure de journalisme (ESJ) de Lille en pleine évolution et à l'apparente baisse d'intérêt des entreprises de presse pour les formations reconnues dans la Convention collective. On peut cependant sans aucun doute, faire confiance à Jean-Marie Charon, observateur particulièrement vigilant et aguerri de la presse, pour ne pas être l'otage d'un système qu'il a, jusqu'ici, analysé avec la plus grande finesse.

\section{NOTES}

1. L'ISIC a organisé l'un des tout premiers en novembre 1990 sous le titre d'«Ethique et Communication ». Les actes ont été publiés par le GREC/O en 1991. On peut se les procurer auprès de Communication et Organisation (200F).

2. Groupe de réflexion lancé dans le cadre de l'Université d'Eté de la Communication par Philippe Lucas, sociologue, membre du Comité National d'Ethique et Marcel Des vergne, fondateur du réseau des Universités de la Communication, et qui a été à l'origine de plusieurs colloques sur « Ethique et Information » (1991, 92, 93 et 94) et de l'embryon d'un Observatoire des Pratiques Journalistiques (!) dirigé par Patrick Champagne. Ont participé aux travaux du Groupe de Carcans professionnels de l'information, chercheurs et représentants du monde de la société civile: Ronny Abraham, Yves Agnès, Marie-Françoise Bechtel, Jean-Marie Borzeix, Pierre Bourdieu, Françoise Boursier, Paul Boury, Roland Cayrol, Patrick Champagne, Jean-Marie Charon, Claire Chazal, JeanPierre Delivet, François Henri de Vineu, Franqois d'Orcival, Jean-Marie Dupont, Catherine Ehrel, Jean-Pierre Farkas, Thomas Ferenczi, Michel Garin, Jacques Gonnet, Hervé Guilbaud, Serge Hirel, Daniël Junqua, JeanFrançois Kahn, 
3. Philippe Loquay, Manuel Lucbert. Jean Martin, Micheline Orlernans, lacques Paty, Patrick Pépin. Clément Pieuchot, Icannette Rabau Daudon, Jean-Louis Rollot. Dommique Wolton. 3 33, rue du Louvre 75002 Paris Tél (1) 44822081

4. Extraits de la plaquette de présentation du programme

5. Idem

6. Idem

7. La Presse en France de 1945 à nos jours. Paris, Seuil. 